\title{
Identificazione molecolare di mutazioni conferenti resistenza a rifampicina ed isoniazide in $M$. tuberculosis in campioni clinici diretti mediante Genotype MTBDR (Hain Lifescience)
}

\author{
Paolo Miotto', Federica Piana', Valeria Penati' ${ }^{2}$, Luigi Ruffo Codecasa², Daniela Maria Cirillo' \\ 'Unità Patogeni Batterici Emergenti, Istituto Scientifico San Raffaele, Milano \\ 'Istituto Villa Marelli, Ospedale Niguarda Ca' Granda, Milano
}

Key words: MDR-TB, Genotype MTBDR, LiPA Assay

Identification of mutations conferring rifampin and isonizid resistance in clinical samples using the Genotype MTBDR (Hain Lifescience)

\section{SUMMARY}

The widespread of multi-drug resistant (MDR) Mycobacterium tuberculosis, resistant at least to rifampin (RIF) and isoniazid (INH), represents serious threats to the control of tuberculosis (TB) and increases the public health challenge worldwide. Surveillance program and rapid identification of drug-resistance strains are key-elements for an early and appropriate TB management.

The aim is to evaluate the Genotype MTBDR (Hain Lifescience, Nehren, Germany), a reverse hybridization-based assay, as a rapid tool to identify mutations in the $r p o B$ and katG genes associated to RIF-/INH-resistance directly in clinical specimens. We also evaluate the performance of a paper based device (Genocard - Hain Lifescience, Nehren, Germany) to collect and transport inactivated biological material.

The test was evaluated retrospectively on 68 respiratory samples with positive cultures for M. tuberculosis. Considering the smear-positive samples only, the Genotype MTBDR gave interpretable results in 56 out of 57 samples (98.2\%). The main limitations of the Genotype MTBDR are the difficulties in the amplification from smear-negative samples and the low sensitivity for the INH-resistance. The inclusion of probes targeting other regions involved in INH-resistance will increase the sensitivity of the test.

The GenoCard, with its easy- and rapid-to use features, represents a functional tool for the sample collection with cost-effective and bio-safety benefits. The possibility to use the GenoCard directly in amplification reactions facilitates the gathering of data by molecular approaches.

\section{INTRODUZIONE}

L'insorgenza e la diffusione di ceppi di Mycobacterium tuberculosis (MTB) farmaco-resistenti rappresentano un notevole ostacolo ai programmi di controllo della tubercolosi (TBC) nel mondo (27). I ceppi di MTB multi-resistenti (MDR), resistenti a rifampicina (RIF) e isoniazide (INH), richiedono infatti una terapia antibiotica più lunga e costosa e con minor probabilità di successo terapeutico rispetto ai ceppi sensibili (8). La sorveglianza e la rapida identificazione della farmaco-resistenza nei ceppi sono fondamentali per l'inizio tempestivo di un trattamento terapeutico adeguato evitandone/limitandone la diffusione.

La determinazione di farmaco-resistenza mediante $\mathrm{i}$ metodi convenzionali (coltura ed antibiogramma sui ceppi isolati) richiedono tempi lunghi anche utilizzando i sistemi di coltura liquidi; pertanto, vi è la necessità di sviluppare saggi semplici, rapidi e costo-efficaci basati su tecniche molecolari utilizzabili in micobatteriologia clinica per l'individuazione di mutazioni genomiche responsabili di farmaco-resistenza (7). I test molecolari rappresentano uno strumento potenzialmente utile in Paesi dove l'assenza di laboratori in grado di allestire colture micobatteriche o la loro localizzazione non permette una raccolta dati adeguata circa la diffusione di ceppi MDR. Inoltre, l'approccio genotipico renderebbe possibile una previsione sulla risposta alla terapia $(5,15,22)$.

La resistenza alla RIF è dovuta a mutazioni a carico di una regione relativamente ristretta di circa $80 \mathrm{bp}$ del gene $r p o B$ che codifica la subunità $\beta$ dell'RNA polimerasi $(21,24)$. La resistenza all'INH è invece causata da mutazioni a carico di molteplici regioni geniche tra le quali kat $G$, inhA e $n d h(2,13,18,19,21,23,26)$. Le mutazioni a carico del codone 315 del gene kat $G$ rappresentano il $60-80 \%$ dei casi INH-resistenti (INH-R). Sono state identificate mutazioni anche nelle regioni regolatorie di inhA e $\operatorname{ahpC}$-oxyR $(3,9,11)$. L'analisi di ceppi di MTB INH-R isolati in diver- 
si Paesi ha dimostrato che la prevalenza di tali mutazioni varia su scala geografica (16).

Il Genotype MTBDR (Hain Lifescience, Nehren, Germany) è un test recentemente sviluppato per l'identificazione di RIF- e/o INH-resistenza in ceppi di MTB $(6,10)$. Il test si basa sull'ibridizzazione inversa tra prodotti di amplificazione derivati da una PCR multiplex e sonde oligonucleotidiche fissate su supporto di nitrocellulosa complementari a regioni wild-type (WT) dei geni $r p o B$ e $k a t G$ e alle principali mutazioni che interessano i due geni. L'utilizzo del test per l'identificazione di mutazioni associate a RIF- e INHresistenza in ceppi di MTB ha mostrato valori di specificità superiori al $99 \%$ e di sensibilità del $98 \%$ per RIF-R e del $70-90 \%$ per INH-R $(6,10)$. Lo scopo del presente studio è valutare il Genotype MTBDR come strumento per l'identificazione rapida di mutazioni nei geni rpoB e kat $G$ associate a RIF- e INH-resistenza, rispettivamente, in campioni clinici diretti. A questo proposito, il DNA estratto da 68 campioni respiratori MTD positivi (bioMerieux, Marcy 1'Etoile, France) provenienti da pazienti con alto sospetto di TBC attiva è stato analizzato con il Genotype MTBDR. Per la sorveglianza delle farmaco-resistenze su ampia scala anche in Paesi in cui la coltura non è eseguita in routine, si sta valutando la possibilità di utilizzare un supporto (GenoCard - Hain Lifescience, Nehren, Germany) per la raccolta e il trasporto sicuro di materiale biologico inattivato da utilizzare direttamente in test molecolari per la determinazione di farmaco-resistenze e genotipizzazione.

\section{MATERIALI E METODI}

Campioni clinici ed estrazione del DNA. In questo studio prospettico, 68 campioni respiratori MTD positivi (bioMerieux, Marcy l'Etoile, France) provenienti da pazienti con alto sospetto di TBC attiva sono stati raccolti presso il Centro di Riferimento Regionale per i micobatteri "Villa Marelli".

Per l'estrazione del DNA genomico dai campioni clinici, $500 \mu \mathrm{L}$ di campione decontaminato sono stati centrifugati a $13.000 \mathrm{rpm}$ per $15 \mathrm{~min}$; il pellet è stato risospeso in $75 \mu \mathrm{L}$ di acqua sterile distillata e i micobatteri sono stati lisati mediante incubazione a $95^{\circ} \mathrm{C}$ per $30 \mathrm{~min}$ e sonicazione per $15 \mathrm{~min}$. Le provette sono state centrifugate a $13.000 \mathrm{rpm}$ per 10 min a $4^{\circ} \mathrm{C}$ e $5 \mu \mathrm{L}$ di surnatante sono stati utilizzati per l'allestimento di reazioni di amplificazione.

I campioni clinici sono stati inoltre inoculati per la crescita dei micobatteri su terreno liquido MGIT 9600 (BD, Cockeysville, MD). La sensibilità a RIF ed INH sui ceppi di MTB è stata valutata mediante MGIT e metodo proporzionale su Löwenstein-Jensen (LJ).
La classificazione dei 68 campioni attraverso l'analisi microscopica e il profilo di sensibilità a RIF e INH sono riassunti in Tabella 1.

GenoCard. Il supporto solido GenoCard (Hain Lifescience, Figura I) permette il trasporto di campioni clinici inattivati e può essere utilizzato direttamente in una reazione di amplificazione. L'utilizzo del supporto è stato valutato impiegando 20 campioni clinici. Per l'estrazione del DNA genomico, $100 \mu \mathrm{L}$ di campione decontaminato sono stati posti sul supporto, lasciati essiccare a temperatura ambiente per 2 ore ed inattivati mediante incubazione a $110^{\circ} \mathrm{C}$ per $15 \mathrm{~min}$. Un talloncino ottenuto con lo strumento fornito dal kit (ø $1 \mathrm{~mm} \mathrm{ca}$.) di GenoCard è stato utilizzato direttamente per l'allestimento della reazione di amplificazione. I campioni biologici sono stati inoltre inoculati per la crescita di MTB e la sensibilità a RIF e INH dei ceppi isolati è stata valutata mediante MGIT e metodo proporzionale su LJ. Amplificazione. Sono stati utilizzati $5 \mu \mathrm{L}$ di surnatante per l'allestimento delle reazioni di amplificazione con i primers biotinilati forniti. L'amplificazione è stata eseguita mediante il termociclatore Gene-Amp System 2400 (PerkinElmer) utilizzando $1 \mathrm{U}$ di HotStarTaq DNA Polymerase (Qiagen) in un volume finale di 50 $\mu \mathrm{L}$. Il protocollo di amplificazione prevede un ciclo a $95^{\circ} \mathrm{C}$ per $15 \mathrm{~min}$ (attivazione Taq), 10 cicli di denaturazione a $95^{\circ} \mathrm{C}$ per $30 \mathrm{sec}$ e primerannealing a $58^{\circ} \mathrm{C}$ per 2 min, 40 cicli di denaturazione a $95^{\circ} \mathrm{C}$ per $25 \mathrm{sec}$, primer-annealing a $53^{\circ} \mathrm{C}$ per $40 \mathrm{sec}$ ed estensione a $70^{\circ} \mathrm{C}$ per $40 \mathrm{sec}$ e un ciclo di estensione finale a $70^{\circ} \mathrm{C}$ per $8 \mathrm{~min}$.

Per l'impiego del supporto GenoCard, sono state utilizzate $2 \mathrm{U}$ di HotStarTaq DNA Polymerase (Qiagen) e il medesimo protocollo di amplificazione. La PCR multiplex produce amplificati delle dimensioni di $200 \mathrm{bp}$ (controllo universale UC specie-specifico), 170 bp (frammento di rров) e 120 bp (frammento di katG). La dimensione dei prodotti di amplificazione è stata verificata su gel di agarosio al $2 \%$ come riportato sul manuale d'uso del test.

Test Genotype MTBDR. Il test Genotype MTBDR (Hain Lifescience) permette l'identificazione di mutazioni conferenti RIF-R e INH-R. Il test si basa sull'ibridizzazione inversa tra prodotti di amplificazione derivati da una PCR multiplex e sonde oligonucleotidiche fissate su supporto di nitrocellulosa complementari a regioni wildtype (WT) sovrapposte delle regioni d'interesse (rpoB WT1-rpoB WT5, katG WT), le quattro mutazioni più ricorrenti in rpoB (sonde $r p o B$ MUT), e mutazioni al codone 315 di kat $G$ (sonde $k a t G$ MUT) (Figura II). Sono presenti un controllo universale (UC) per verificare la presenza di 
amplificati derivati da batteri Gram-positivi con alto contenuto G-C e un controllo specifico (TUB) per MTB complex che ha come bersaglio il gene che codifica il 23S rRNA. Dopo denaturazione, gli amplificati single-stranded biotinilati vengono ibridizzati alle sonde oligonucleotidiche fissate su nitrocellulosa $\left(45^{\circ} \mathrm{C}\right.$ per $\left.30 \mathrm{~min}\right)$. Successivamente le strisce vengono lavate per 15 min a $45^{\circ} \mathrm{C}$, come riportato sul manuale d'uso. L'aggiunta di coniugato streptavidina/fosfatasi alcalina (AP) e di un substrato enzimatico cromogeno specifico per la AP permette di rilevare l'ibridizzazione. I risultati sono disponibili in una giornata lavorativa, con un unico ciclo di PCR. L'ibridizzazione e i lavaggi sono stati eseguiti con uno strumento automatizzato (APOLLO 20/2TC; MATEC Medizintecnik GmbH, Munsingen, Germany) o manualmente utilizzando l'ibridizzatore TWINCUBATOR (Hain Lifescience, Nehren, Germany). Le strisce sono state interpretate per la sensibilità o resistenza a RIF ed INH secondo quanto riportato dal manuale d'uso (Figura II).

\section{RISULTATI}

L'analisi microscopica in fluorescenza per i 68 campioni clinici respiratori utilizzati nello studio, definita secondo gli standard OMS, ha permesso di identificare 27 campioni negativi o debolmente positivi, 1 campione,+ 35 campioni ++ e 5 campioni +++ (Tabella 1$)$.

La sensibilità a RIF e INH dei ceppi isolati dall' $i-$ noculo dei campioni in coltura è stata valutata mediante MGIT e confermata con il metodo proporzionale su LJ (28). I risultati ottenuti dal test molecolare sono stati confrontati con i risultati dei test fenotipici.

Il test molecolare ha prodotto 9 differenti profili di ibridizzazione, riportati in Tabella 1 . Quarantasei campioni sono stati classificati sensibili a RIF e INH dal test, 7 campioni hanno mostrato un profilo di ibridizzazione che individua la presenza di specifiche mutazioni in $r p o B$ e/o kat $G$ e in 3 casi l'interpretazione della striscia è risultata difficoltosa a causa di un segnale di ibridizzazione debole o non interpretabile per alcune bande (rpoB WT bande 3 o 5) o per un doppio profilo d'ibridizzazione per katG (presenza di segnale WT e mutato simultanei).

L'antibiogramma per i 46 campioni classificati come sensibili dal test molecolare, ha confermato la sensibilità alla RIF in tutti i campioni (46/46) e in $45 / 46$ casi per INH. Un campione classificato come sensibile dal test MTBDR è risultato INH$\mathrm{R}$ con $\mathrm{i}$ test tradizionali.

Il test molecolare ha identificato correttamente 3 campioni MDR, confermati dall'antibiogramma. In un caso il test MTBDR ha identificato sola- mente la rifampincino-resistenza mentre l'analisi fenotipica ha confermato la presenza di un ceppo MDR evidenziando resistenza anche a INH.

Quattro campioni sono stati riconosciuti come INH-R dal test mediante l'identificazione della mutazione AGC315ACC nel gene katG (2 dei quali mediante la presenza di doppio profilo di ibridizzazione per le sonde WT-MUT). Tutti i risultati ottenuti mediante l'analisi genotipica sono stati confermati dall'antibiogramma.

La funzionalità del supporto GenoCard per il trasporto, la conservazione a medio-termine (fino a 6 mesi) di campioni biologici contenenti DNA micobatterico e il suo impiego in saggi che sfruttano l'amplificazione degli acidi nucleici sono stati valutati utilizzando il test Genotype MTDBR. Il DNA genomico proveniente da 20 campioni clinici diretti è stato estratto da $100 \mu \mathrm{L}$ di materiale biologico decontaminato posti sul supporto, come descritto nei Materiali e Metodi. Un talloncino (ø $1 \mathrm{~mm}$ ca.) di GenoCard è stato utilizzato direttamente per l'allestimento della reazione di PCR multiplex impiegata dal test MTBDR. La sensibilità a RIF e INH dei ceppi di MTB isolati dall'inoculo dei campioni è stata valutata mediante MGIT e metodo proporzionale su LJ. I risultati ottenuti dal test molecolare sono stati confrontati con i risultati dei test fenotipici.

Per 14 dei 20 campioni è stato possibile ottenere i prodotti di amplificazione attesi mentre per 6 sedimenti $(5 / 6$ risultati negativi o debolmente positivi alla microscopia) l'amplificazione non ha permesso di ottenere i prodotti attesi. I risultati di ibridizzazione ottenuti sono riportati in Tabella 2. Il test di sensibilità eseguito con il MGIT per i 14 campioni classificati sensibili dal test molecolare, ha confermato la sensibilità in tutti questi per quanto riguarda la RIF e in 12 casi per l'INH. Un campione classificato come sensibile dal test MTBDR ha originato in coltura un ceppo INH-R.

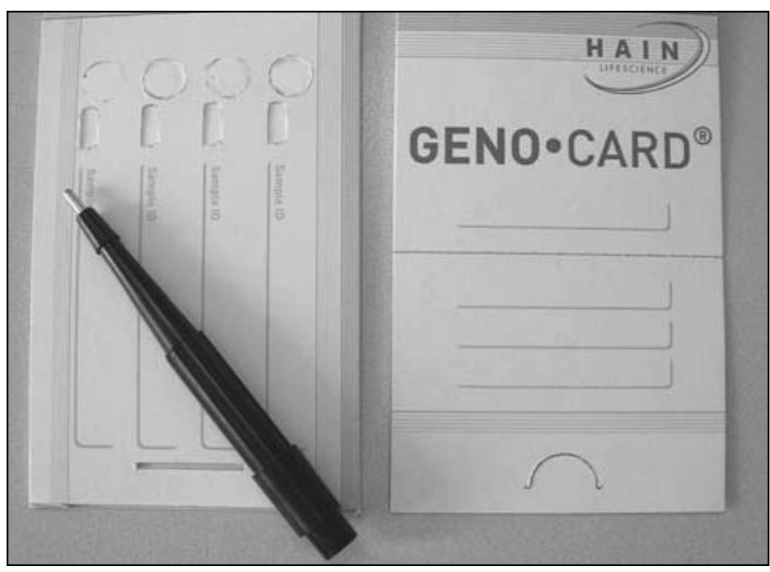

Figura I. Supporto solido GenoCard. II supporto è costituito da apposito materiale in grado di trattenere gli inibitori della reazione di amplificazione. 


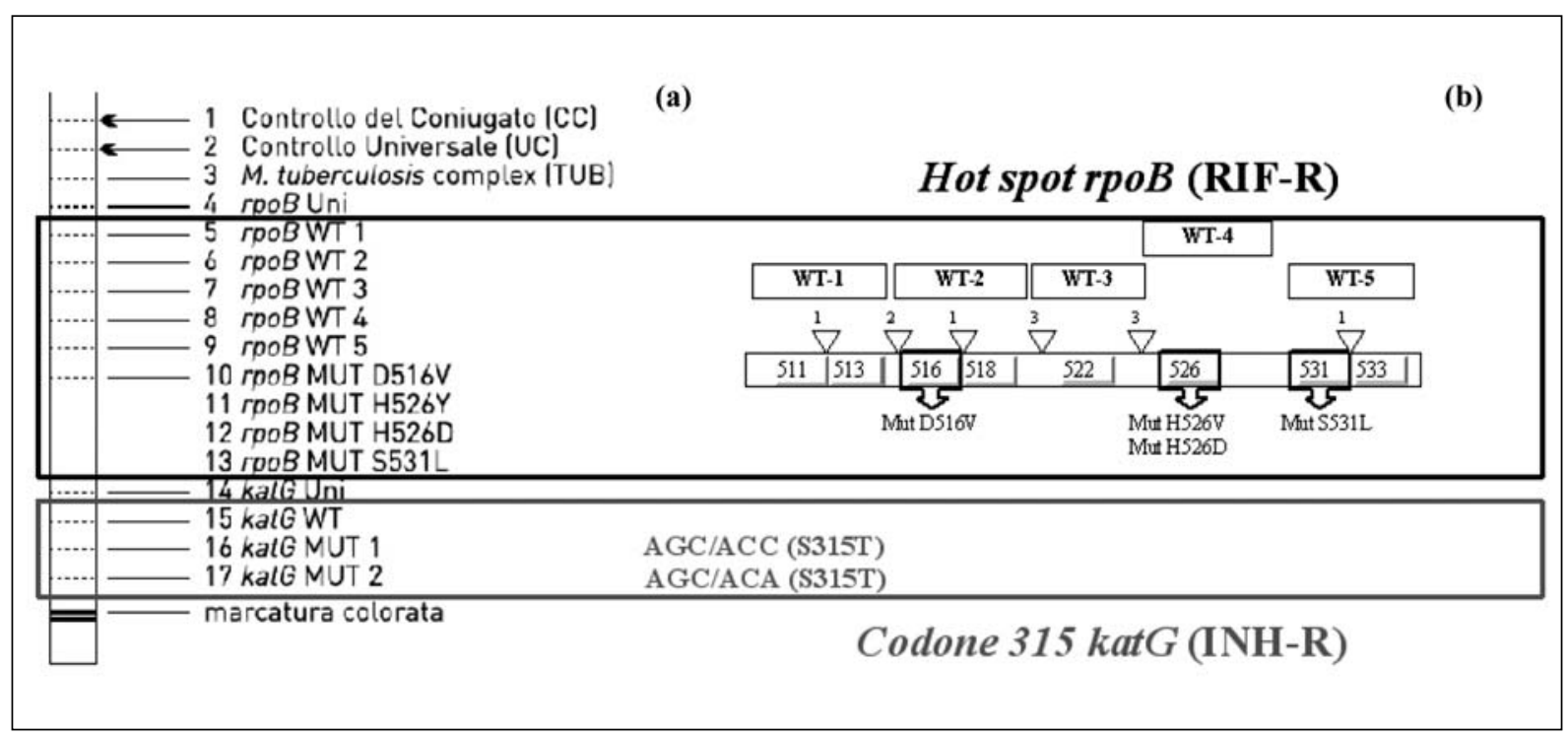

Figura II. (a) Rappresentazione di una striscia indicante la posizione delle sonde wild-type e mutate. La presenza di mutazione è indicata dalla mancanza di ibridizzazione di una o più sonde WT con o senza l'ibridizzazione di sonde MUT. Sono presenti inoltre un controllo universale (UC) specifico per l'amplificazione di DNA derivato da batteri gram-positivi con alto contenuto $G+C$ e un controllo specifico (TUB) per isolati appartenenti al M. tuberculosis complex.

(b) Rappresentazione schematica della regione hot spot di rpoB riportante le posizioni delle sonde WT e MUT presenti nella striscia. La lunghezza media delle sonde oligonucleotidiche è di 20 basi. Le frecce indicano il numero e la posizione dei codoni omessi.

Tabella I. Risultati dell'antibiogramma e del test molecolare Genotype MTBDR per l'identificazione di mutazioni conferenti resistenza a RIF e INH in 68 campioni clinici diretti.

L'analisi microscopica è stata definita secondo gli standard OMS: negativo, non si osservano bacilli alcol-acido resistenti (BAAR); debolmente positivo, I-9 BAAR in 100 campi; +, I0-99 BAAR in 100 campi; ++, I-10 BAAR per campo in almeno 10 campi; +++, >10 BAAR per campo in almeno 10 campi. In grassetto sono riportati i 3 casi misinterpretati dovuti ad un segnale d'ibridizzazione debole per alcune bande (rpoB (presenza di ibridizzazione per entrambe le sonde WT e MUT). I campioni marcati (*) sono stati correttamente definiti dalla versione Genotype MTBDR plus.

\begin{tabular}{|c|c|c|c|c|c|c|}
\hline \multirow{3}{*}{ BAAR } & \multirow{3}{*}{ Antibiogramma } & \multicolumn{3}{|c|}{ Genotype MTBDR } & \multirow{3}{*}{$\begin{array}{c}\text { No. } \\
\text { Campioni } \\
\text { clinici }\end{array}$} & \multirow{3}{*}{$\%$} \\
\hline & & & & katG & & \\
\hline & & WT & MUT & katG & & \\
\hline \multirow[t]{8}{*}{ Debolm. positivo } & MDR & $1,2,4$ & 1 & TI & 1 & 1.5 \\
\hline & & 2,4 & 1 & $\mathrm{TI}$ & 1 & 1.5 \\
\hline & INH-R & $\mathrm{I}, 2,3,4,5$ & 1 & $\mathrm{TI}$ & $\mathrm{I}$ & 1.5 \\
\hline & & Non ampl. & & & 1 & 1.5 \\
\hline & $S$ & $\mathrm{I}, 2,3,4,5$ & 1 & WT & 11 & 16.2 \\
\hline & & Non ampl. & & & 10 & 14.7 \\
\hline & & $1,2,4 *$ & & WT & 1 & 1.5 \\
\hline & & $1,2,3,4^{*}$ & & WT & $\mathrm{I}$ & 1.5 \\
\hline+ & MDR & $1,2,3,4$ & S53IL & WT & 1 & 1.5 \\
\hline \multirow[t]{6}{*}{++} & MDR & $1,2,3,4,5$ & S53IL & WT-TI & 1 & 1.5 \\
\hline & INH-R & $1,2,3,4,5$ & 1 & WT & 1 & 1.5 \\
\hline & & $1,2,3,4,5$ & 1 & WT-TI & 2 & 2.9 \\
\hline & S & $1,2,3,4,5$ & 1 & WT & 29 & 42.6 \\
\hline & & $1,2,3,4,5$ & 1 & WT-TI $^{+}$ & 1 & 1.5 \\
\hline & & Non ampl. & & & 1 & 1.5 \\
\hline+++ & $\mathrm{S}$ & $1,2,3,4,5$ & 1 & WT & 5 & 7.4 \\
\hline
\end{tabular}


Tabella II. Risultati dell'antibiogramma e del test molecolare Genotype MTBDR per l'identificazione di mutazioni conferenti resistenza a RIF e INH in 20 campioni clinici diretti utilizzando il supporto solido GenoCard. In grassetto è riportato il campione INH-R non identificato dal test molecolare.

\begin{tabular}{|c|c|c|c|c|c|c|}
\hline \multirow{3}{*}{ BAAR } & \multirow{3}{*}{ Antibiogramma } & \multirow{3}{*}{ Materiale } & \multicolumn{3}{|c|}{ Genotype MTBDR } & \multirow{3}{*}{$\begin{array}{c}\mathbf{N}^{\circ} \text { campioni } \\
(\%)\end{array}$} \\
\hline & & & \multicolumn{2}{|c|}{ rpoB } & \multirow{2}{*}{ KatG } & \\
\hline & & & WT & MUT & & \\
\hline \multirow[t]{5}{*}{ NEG } & $S$ & Agoaspirato & Non ampl. & & & I (5) \\
\hline & & Broncoaspirato & Non ampl. & & & I (5) \\
\hline & & Dren. Ascesso & Non ampl. & & & $\mathrm{I}(5)$ \\
\hline & & Esp. Indotto & Non ampl. & & & I (5) \\
\hline & & Espettorato & $1,2,3,4,5$ & - & WT & I (5) \\
\hline \multirow[t]{2}{*}{ Debolm. positivo } & $S$ & Espettorato & Non ampl. & & & I (5) \\
\hline & & & $1,2,3,4,5$ & - & WT & I (5) \\
\hline \multirow[t]{6}{*}{++} & $\mathrm{S}$ & Broncoaspirato & Non ampl. & & & I (5) \\
\hline & & & $1,2,3,4,5$ & - & WT & $2(10)$ \\
\hline & & Esp. indotto & $1,2,3,4,5$ & - & WT & I (5) \\
\hline & & Espettorato & $1,2,3,4,5$ & - & WT & $5(25)$ \\
\hline & INH-R & Escreato & $1,2,3,4,5$ & - & WT & I (5) \\
\hline & & Espettorato & $1,2,3,4,5$ & - & $\mathrm{TI}$ & I (5) \\
\hline+++ & $\mathrm{S}$ & Espettorato & $\mathrm{I}, 2,3,4,5$ & - & WT & $2(10)$ \\
\hline
\end{tabular}

\section{DISCUSSIONE}

La sempre maggiore diffusione di ceppi di MTB resistenti ai farmaci antitubercolari rende cruciale l'identificazione rapida delle resistenze per instaurare tempestivamente un trattamento adeguato e limitarne, quindi, la diffusione. I metodi convenzionali richiedono ancora almeno 2 settimane, prima di rendere disponibile il risultato dell'antibiogramma. In questo studio abbiamo valutato l'impiego del test Genotype MTBDR per identificare rapidamente RIF- e INH-resistenza in MTB in campioni clinici diretti. RIF e INH sono farmaci chiave nel trattamento della TBC. La resistenza alla RIF è raramente osservata come mono-resistenza e, per questa ragione, è spesso considerata come marcatore per ceppi MDR (21). Diversi studi riportati in letteratura sottolineano l'esistenza di una correlazione tra specifiche mutazioni genomiche e MIC (Minimum Inhibitory Concentration) ed evidenziano come specifiche mutazioni siano responsabili di crossresistenza tra farmaci $(5,12,13,15,22,25)$. L'INH è utilizzata per il trattamento dell'infezione tubercolare latente nei contatti di caso infettivo. Per queste ragioni è critico per il clinico conoscere tempestivamente la sensibilità di un determinato ceppo di MTB a questi due farmaci.

Il test Genotype MTBDR permette l'identificazione rapida della maggior parte delle mutazioni responsabili di RIF- e INH-resistenza in MTB, utilizzando sonde oligonucleotidiche per l'ibridizzazione con le regioni hot-spot di rpoB e il codone 315 di katG. Mutazioni che interessano questo codone sono responsabili di INH-resistenza in circa il $60 \%$ dei casi a livello mondiale $(1,16)$.
Il test Genotype MTBDR è stato eseguito direttamente da campioni clinici per identificare rapidamente pazienti infettati da ceppi di MTB resistenti a RIF e/o INH. È stato possibile ottenere un amplificato specifico in 56 dei 68 campioni PCR positivi (MTD). Alcuni campioni risultati negativi all'analisi microscopica hanno prodotto un profilo di amplificazione non utilizzabile per il test di ibridizzazione. Escludendo i campioni negativi o debolmente positivi all'esame microscopico, è stato possibile eseguire il test molecolare con successo su 56 dei 57 campioni (98.2\%).

Per quanto riguarda la resistenza alla RIF, tutti i campioni sono stati identificati correttamente dal test MTBDR. Alcuni campioni potrebbero essere erroneamente considerati RIF-R per la mancanza di un segnale d'ibridizzazione sufficientemente intenso per le sonde wt; in tal caso è richiesta esperienza per una corretta interpretazione. In uno studio condotto su soli campioni sputo-positivi, anche Somoskovi et al. (20), hanno riscontrato come l'intensità delle bande di ibridizzazione possa variare.

Per i campioni INH-R, mutazioni in katG sono state identificate dal test nel $75 \%(6 / 8)$ dei casi. La scarsa sensibilità del test per l'identificazione di INH-R è dovuta alla mancanza di sonde che hanno come target altri geni implicati nella resistenza all'INH. L'aggiunta di sonde per l'identificazione di mutazioni in questi geni e nella regione dell'operone mabAF-inhA, porterebbe ad un aumento del $15-20 \%$ dei casi INH-R identificati dal test. In un caso è stato riscontrato un doppio profilo di ibridizzazione non confermato dall'antibiogramma. 
Come riportato in letteratura, vi sono ceppi con bassi livelli di resistenza alla RIF non rilevabili dai test fenotipici di routine eseguiti su Löwenstein-Jensen con una concentrazione di farmaco nel terreno di coltura pari a $40 \mu \mathrm{g} / \mathrm{mL}$. Questi bassi livelli di resistenza sono dovuti alla presenza di popolazioni etero-alleliche (wt-mut) con specifiche mutazioni in $r p o B$ che i line probe assay sono in grado di identificare (22). La sensibilità del test Genotype MTBDR riferita ai 41 campioni positivi all'analisi microscopica è del 97.6\% (40/41) per la RIF-R e 90.2\% (37/41) per la INH-R. Valori simili sono stati riscontrati per studi analoghi su campioni clinici diretti positivi alla microscopia $(4,20)$.

Tre pazienti affetti da TBC MDR inclusi nello studio sono stati correttamente identificati dal test molecolare. Considerando la presenza di mutazioni conferenti alti livelli di RIF-R (come la sostituzione S531L) come marcatore di MDR-TB, il test è stato in grado di identificare correttamente tutti i pazienti MDR inclusi nello studio.

Approcci genetici che hanno come target i geni rpoB e katG sono utili strumenti per l'identificazione rapida di casi MDR in popolazioni selezionate. Infatti, diversi studi suggeriscono come la frequenza di particolari mutazioni e la correlazione tra RIF-resistenza e fenotipo MDR possa essere differente in determinate popolazioni $(16,17)$. La principale limitazione del test è rappresentata dai problemi di amplificazione riscontrati per campioni clinici microscopico-negativi e la bassa sensibilità per la resistenza all'INH. L'aggiunta di nuove sonde oligonucleotidiche per l'identificazione di altre mutazioni responsabili di INH-resistenza porterebbe ad un aumento nella sensibilità del test a questa farmaco-resistenza.

Recentemente (Giugno 2007) è stata commercializzata la nuova versione del test (Genotype MTBDRplus). Accanto all'introduzione di nuove sonde per l'identificazione di ulteriori mutazioni responsabili di RIF-R e INH-R (estensione dell'analisi della regione hot spot di $r p o B-\operatorname{cod} .505-$ 510; regione del promotore del gene inhA nucleotidi $-8,-15$ e -16), il nuovo test è stato ottimizzato per migliorarne la sensibilità sui campioni BAAR-negativi e/o debolmente positivi. I campioni oggetto di questo studio sono stati analizzati con il Genotype MTBDRplus. Con il nuovo test è stato possibile ottenere un risultato interpretabile per un ulteriore $13 \%$ dei campioni debolmente positivi rispetto alla precedente versione del test molecolare. In particolare, anche i 2 campioni da cui si era ottenuto un segnale d'ibridizzazione insufficiente, sono stati definiti con un pattern di bande completo e correttamente interpretabile. Il doppio profilo di ibridizzazione per INH-R riscontrato con il Genotype MTBDR in un campione è stato osservato anche con la nuova versione del test. L'analisi mediante sequenziamento ha confermato la presenza di un doppio segnale wt-mut per il codone 315 del gene kat $G$ in questo campione.

La GenoCard, di semplice utilizzo e rapida inattivazione, risulta un valido strumento per il trasporto di campioni clinici riducendo costi e rischio biologico. Il supporto solido GenoCard permette di eseguire il test Genotype MTBDR con le stesse performance ottenute con l'estrazione diretta dei campioni clinici. La possibilità di utilizzare il supporto direttamente in reazione di amplificazione facilita la raccolta dati sulle farmacoresistenze e sulla diffusione di particolari ceppi mediante approccio molecolare e può essere efficacemente utilizzato in quelle regioni dove non è possibile allestire laboratori per la coltura micobatterica.

\section{BIBLIOGRAFIA}

1. Ahmad S, Mokaddas E. Contribution of AGC to ACC and other mutations at codon 315 of the $k a t G$ gene in isoniazid-resistant Mycobacterium tuberculosis isolates from the Middle East. Int J Antimicrob Agents, 2004; 23: 473-8.

2. Banerjee A, Dubnau E, Quemard A, et al. inhA, a gene encoding a target for isoniazid and ethionamide in Mycobacterium tuberculosis. Science, 1994; 263: 227-30.

3. Banerjee A, Sugantino M, Sacchettini JC, Jacobs WR Jr. The mabA gene from the inhA operon of Mycobacterium tuberculosis encodes a 3-ketoacyl reductase that fails to confer isoniazid resistance. Microbiology, 1998; 144(10): 2697-704.

4. Bang D, Andersen ÅB, Thomsen VØ. Rapid genotypic detection of rifampin- and isoniazid-resistant Mycobacterium tuberculosis directly in clinical specimens. J Clin Microbiol, 2006; 44(7): 2605-8.

5. Brossier F, Veziris N, Truffot-Pernot C, Jarlier V, Sougakoff W. Performance of the Genotype MTBDR Line Probe Assay for detection of resistance to rifampin and isoniazid in strains of Mycobacterium tuberculosis with low- and high-Level resistance. J Clin Microbiol, 2006; 44(10): 3659-64.

6. Cavusoglu C, Turhan A, Akinci P, Soyler I. Evaluation of the Genotype MTBDR assay for rapid detection of rifampin and isoniazid resistance in Mycobacterium tuberculosis isolates. J Clin Microbiol, 2006; 44(7): 2338-42.

7. Cheng VCC, Yew WW, Yuen KY. Molecular diagnostics in tuberculosis. Eur J Clin Microbiol Infect Dis, 2005; 24: 711-20.

8. Gupta R, Kim JY, Espinal MA, Caudron JM, Farmer $\mathrm{PE}$, Raviglione MC. Responding to market failures in tuberculosis: a model to increase access to drugs and treatment. Science, 2001; 293: 1049-51.

9. Herrera-León L, Molina T, Saiz P, Saez-Nieto JA, Jimenez MS. New multiplex PCR for rapid detection 
of isoniazid-resistant Mycobacterium tuberculosis clinical isolates. Antimicrob Agents Chemother, 2005; 49: $144-7$.

10. Hillemann D, Weizenegger M, Kubica T, Richter E, Niemann S. Use of the Genotype MTBDR Assay for rapid detection of rifampin and isoniazid resistance in Mycobacterium tuberculosis complex isolates. J Clin Microbiol, 2005; 43(8): 3699-703.

11. Kelley CL, Rouse DA, Morris SL. Analysis of ahpC gene mutations in isoniazid-resistant clinical isolates of Mycobacterium tuberculosis. Antimicrob Agents Chemother, 1997; 41: 2057-8.

12. Lavander C, Globan M, Sievers A, Billman-Jacobe H, Fyfe J. Molecular characterization of isoniazid-resistant Mycobacterium tuberculosis isolates collected in Australia. Antimicrob Agents Chemother, 2005; 49(10): 4068-74.

13. Lee AS, Teo AS, Wong SY. Novel mutations in $n d h$ in isoniazid-resistant Mycobacterium tuberculosis isolates. Antimicrob Agents Chemother, 2001; 45: 2157-59.

14. Marttila HJ, Soini H, Eerola E, et al. A Ser315Thr substitution in $k a t G$ is predominant in genetically heterogeneous multidrug-resistant Mycobacterium tuberculosis isolates originating from the $\mathrm{St}$ Petersburg area in Russia. Antimicrob Agents Chemother, 1998; 42: 2443-5.

15. Moghazeh SL, Pan X, Arain T, Stover C.K, Musser JM, Kreiswirth BN. Comparative antimycobacterial activities of rifampin, rifapentine, and KRM-1648 against a collection of rifampin-resistant Mycobacterium tuberculosis isolates with known rpoB mutations. Antimicrob Agents Chemother, 1996; 40(1): 2655-7.

16. Mokrousov I, Narvskaya O, Otten T, Limeschenko E, Steklova L, Vyshnevskiy B. High prevalence of katG Ser315Thr substitution among isoniazid-resistant Mycobacterium tuberculosis clinical isolates from northwestern Russia, 1996 to 2001. Antimicrob Agents Chemother, 2002; 46: 1417-24.

17. Piatek AS, Telenti A, Megan R, et al. Genotypic Analysis of Mycobacterium tuberculosis in two distinct populations using molecular beacons: implications for rapid susceptibility testing. Antimicrob Agents Chemother, 2000; 44(1): 103-10.

18. Ramaswamy SV, Reich R, Dou SJ, et al. Single Nucleotide Polymorphisms in genes associated with isoniazid resistance in Mycobacterium tuberculosis. Antimicrob Agents Chemother, 2003; 47(4): 1241-50.

19. Slayden RA, Barry CE III. The genetics and biochemistry of isoniazid resistance in Mycobacterium tuberculosis. Microbes Infect, 2000; 2: 659-69.

20. Somoskovi A, Dormandy J, Mmitsani D, Rivenburg J, Salfinger M. Rapid direct detection and susceptibility testing of the Mycobacterium tuberculosis complex for isoniazid and rifampin in smear positive clinical specimens by the PCR-based Genotype MTBDR Assay. J Clin Microbiol, 2006; 44(12): 4459-63.

21. Somoskovi A, Parsons LM, Salfinger M. The molecular basis of resistance to isoniazid, rifampin, and pyrazinamide in Mycobacterium tuberculosis. Resp Res, 2001; 2: 164-8.

22. Srivastava K, Das R, Jakhmola P, et al. Correlation of mutations detected by INNO-LiPA with levels of rifampicin resistance in Mycobacterium tuberculosis. Indian J Med Res, 2004; 120: 100-5.

23. Telenti A, Honoré N, Bernasconi C, et al. Genotypic assessment of isoniazid and rifampin resistance in Mycobacterium tuberculosis: a blind study at reference laboratory level. J Clin Microbiol, 1997; 35: 719-23.
24. Telenti A, Imboden P, Marchesi F, et al. Detection of rifampicin-resistance mutations in Mycobacterium tuberculosis. Lancet, 1993; 341: 647-50.

25. Uzun M, Erturan Z, Ang Ö. Investigation of crossresistance between rifampin and rifabutin in Mycobacterium tuberculosis complex strains. Int $\mathbf{J}$ Tuberc Lung Dis, 2002; 6(2): 164-5.

26. Vilchéze C, Weisbrod TR, Chen B, et al. Altered $\mathrm{NADH} / \mathrm{NAD}^{+}$ratio mediates coresistance to isoniazid and ethionamide in mycobacteria. Antimicrob. Agents Chemother, 2005; 49: 708-20.

27. World Health Organization/International Union Against Tuberculosis and Lung Disease Global Project on Anti-Tuberculosis Drug Resistance Surveillance. Anti-tuberculosis drug resistance in the world: report no. 3. Geneva, Switzerland: World Health Organization; 2004.

28. World Health Organization. Guidelines for surveillance of drug resistance in tuberculosis. Geneva, WHO/CDS/TB/2003.32, 30-1.
Daniela Maria Cirillo

MD PhD - Unità Patogeni Batterici Emergenti San Raffaele-Turro

Via Stamira d'Ancona 20, 20127 Milano

Tel.: 0226437947 - Fax: 0226437989

E-mail: cirillo.daniela@hsr.it 